\title{
Correction: Ratnayake et al. Assessment of Breast Cancer Surgery in Manitoba: A Descriptive Study. Curr. Oncol. 2021, $28,581-592$
}

\author{
Iresha Ratnayake ${ }^{1,2, *}$, Pamela Hebbard ${ }^{3,4} \mathbb{D}^{\mathbb{D}}$, Allison Feely ${ }^{1}$, Natalie Biswanger ${ }^{2,5}$ and Kathleen Decker ${ }^{1,2,6}{ }^{\mathbb{D}}$ \\ 1 Department of Epidemiology \& Cancer Registry, CancerCare Manitoba, Winnipeg, MB R3E 0V9, Canada; \\ afeely@cancercare.mb.ca (A.F.); kdecker@cancercare.mb.ca (K.D.) \\ 2 Department of Community Health Sciences, Rady Faculty of Health Sciences, University of Manitoba, \\ Winnipeg, MB R3E 3P5, Canada; nbiswanger@cancercare.mb.ca \\ 3 Department of Surgery, Rady Faculty of Health Sciences, University of Manitoba, \\ Winnipeg, MB R3E 3P5, Canada; phebbard@cancercare.mb.ca \\ 4 CancerCare Manitoba, Winnipeg, MB R3E 0V9, Canada \\ 5 Screening Programs, CancerCare Manitoba, Winnipeg, MB R3C 2B1, Canada \\ 6 Research Institute in Oncology \& Hematology, CancerCare Manitoba, Winnipeg, MB R3E 0V9, Canada \\ * Correspondence: iratnayake@cancercare.mb.ca; Tel.: +1-20-47-842781
}

check for updates

Citation: Ratnayake, I.; Hebbard, P.; Feely, A.; Biswanger, N.; Decker, K. Correction: Ratnayake et al. Assessment of Breast Cancer Surgery in Manitoba: A Descriptive Study. Curr. Oncol. 2021, 28, 581-592. Curr. Oncol. 2021, 28, 2775-2777. https:// doi.org/10.3390/curroncol28040242

Received: 1 July 2021

Accepted: 5 July 2021

Published: 20 July 2021

Publisher's Note: MDPI stays neutral with regard to jurisdictional claims in published maps and institutional affiliations.

Copyright: ( $\odot 2021$ by the authors. Licensee MDPI, Basel, Switzerland. This article is an open access article distributed under the terms and conditions of the Creative Commons Attribution (CC BY) license (https:// creativecommons.org/licenses/by/ $4.0 /)$.
The authors wish to make a correction to this paper due to a minor change in indicator definition [1]. The overall findings of the study remain unchanged; however, we wish to include the updated data in the manuscript.

In the original article, the abstract stated that the axillary lymph node dissection for node-negative disease ranged from $11.8 \%$ to $33.3 \%$. The range should be corrected to $3.4 \%$ to $32.6 \%$.

In Section 3.4, the original article stated, "The quality indicators measured are summarized in Table 3. In Manitoba, $19.6 \%$ of women with confirmed node-negative disease received an axillary lymph node dissection. The percentage of women who received ALND for node-negative disease increased with age ( $2.6 \% 95 \%$ CI: 0.0 to 7.7 in $20-29$ vs. $29.3 \% 95 \%$ CI: 19.4 to 39.1 in $80+$ ). The percentage of women who underwent ALND for node-negative disease also varied by RHA of residence at diagnosis. Among women who lived in urban RHA, only $11.8 \%$ (95\% CI: 8.5 to 15.2 ) underwent this procedure compared to a range of $21.0 \%$ (95\% CI: 10.8 to 31.1 ) to $33.3 \%$ (95\% CI: 26.1 to 40.6 ) in rural RHAs. Of those patients who received an axillary dissection for node-negative disease, most had stage I cancer. Among those who had surgery in urban RHA, 13.5\% (95\% CI: 10.6 to 16.4) underwent ALND for node-negative disease compared to 38.0\% (95\% CI: 29.8 to 46.1 ) in rural 1 and $42.4 \%$ (95\% CI: 25.6-59.3) in rural 2".

It should be replaced with the following,

"The quality indicators measured are summarized in Table 3. In Manitoba, 19.6\% of women who underwent an axillary lymph node dissection were node negative. When looking at the percentage of node negative patients who underwent axillary dissection however, $5.8 \%$ of women with confirmed node-negative disease received an axillary lymph node dissection. This number was variable when looking at certain demographic factors. The percentage of women who received ALND for node-negative disease increased with age (1.9\% 95\% CI: 0.0 to 5.7 in 20-39 versus $7.9 \% 95 \%$ CI: 4.9 to 10.9 in $80+)$. The percentage of women who underwent ALND for node-negative disease also varied by RHA of residence at diagnosis. Among women who lived in urban RHA, only 3.0\% (95\% CI: 2.1 to 3.9) underwent this procedure compared to a range of $4.8 \%$ (95\% CI: 2.3 to 7.4$)$ to $15.9 \%$ (95\% CI: 12.0 to 19.8) in rural RHAs. Of those patients who received an axillary dissection for node-negative disease, most had stage II cancer. Among those who had surgery in urban RHA, 3.4\% (95\% CI: 2.6 to 4.2) underwent ALND for node-negative disease compared to $20.4 \%$ (95\% CI: 15.4 to 25.3 ) in rural 1 and $32.6 \%$ (95\% CI: 18.6 to 46.6$)$ in rural 2". 
In the original article, Table 3 was as follows:

Table 3. Surgical quality among women who underwent surgical resection for invasive breast cancer, Manitoba, $2010-2015$.

\begin{tabular}{|c|c|c|c|c|c|c|}
\hline \multirow[t]{2}{*}{ Characteristic } & \multicolumn{2}{|c|}{$\begin{array}{l}\text { Axillary Lymph Node Dissection } \\
\text { for Node Negative Disease }\end{array}$} & \multicolumn{2}{|c|}{$\begin{array}{c}\leq 30 \text { Days Between First Surgical } \\
\text { Consult and First Surgery }\end{array}$} & \multicolumn{2}{|c|}{$\begin{array}{c}\text { Re-excision After } \\
\text { Breast-Conserving Surgery }\end{array}$} \\
\hline & $\mathbf{N}$ & $\%(95 \% \mathrm{CI})$ & $\mathbf{N}$ & $\%(95 \%$ CI $)$ & $\mathbf{N}$ & $\%(95 \%$ CI $)$ \\
\hline Manitoba & 137 & 19.6 & 1245 & 49.3 & 450 & 18.5 \\
\hline \multicolumn{7}{|c|}{ Age Group } \\
\hline $20-39$ & - & $2.6(0.0,7.7)$ & 27 & $32.9(22.8,43.1)$ & 20 & $35.7(23.2,48.3)$ \\
\hline $40-49$ & - & $13.2(7.0,19.4)$ & 176 & $49.4(44.2,54.6)$ & 70 & $24.1(19.1,29.0)$ \\
\hline $50-59$ & - & $12.1(7.1,17.1)$ & 299 & $46.7(42.9,50.6)$ & 118 & $20.0(16.8,23.2)$ \\
\hline $60-69$ & - & $25.0(18.4,31.6)$ & 409 & $54.0(50.4,57.5)$ & 133 & $17.6(14.9,20.3)$ \\
\hline $70-79$ & - & $26.3(18.9,33.6)$ & 227 & $48.6(44.1,53.1)$ & 76 & $15.7(12.5,19.0)$ \\
\hline $80+$ & - & $29.3(19.4,39.1)$ & 107 & $48.0(41.4,54.5)$ & 33 & $12.5(8.5,16.5)$ \\
\hline \multicolumn{7}{|c|}{ RHA of Residence (at diagnosis) } \\
\hline Urban & - & $11.8(8.5,15.2)$ & 766 & $47.9(45.5,50.4)$ & 258 & $17.0(15.1,18.9)$ \\
\hline Rural 1 & - & $33.3(26.1,40.6)$ & 160 & $60.2(54.3,66.0)$ & 73 & $23.5(18.8,28.2)$ \\
\hline Rural 2 & - & $24.0(15.4,32.5)$ & 151 & $49.2(43.6,54.8)$ & 66 & $24.6(19.5,29.8)$ \\
\hline Rural 3 & - & $21.0(10.8,31.1)$ & 150 & $49.8(44.2,55.5)$ & 42 & $14.7(10.6,18.8)$ \\
\hline Rural 4 & - & $23.5(3.4,43.7)$ & 18 & $33.3(20.8,45.9)$ & 11 & $19.3(9.1,29.5)$ \\
\hline \multicolumn{7}{|c|}{ RHA of Surgery } \\
\hline Urban 1 & 71 & $13.5(10.6,16.4)$ & & & 376 & $17.5(15.9,19.1)$ \\
\hline Rural 1 & 52 & $38.0(29.8,46.1)$ & & & 57 & $23.0(17.7,28.2)$ \\
\hline Rural 2 & 14 & $42.4(25.6,59.3)$ & & & & \\
\hline Rural 2-4 & $\mathrm{n} / \mathrm{a}$ & $\mathrm{n} / \mathrm{a}$ & $\mathrm{n} / \mathrm{a}$ & $\mathrm{n} / \mathrm{a}$ & 15 & $46.8(29.6,64.2)$ \\
\hline \multicolumn{7}{|c|}{ Stage } \\
\hline Stage I & 80 & $88.9(82.4,95.4)$ & 609 & $49.6(46.8,52.4)$ & 208 & $15.5(13.6,17.4)$ \\
\hline Stage II & 57 & $18.6(14.3,23.0)$ & 496 & $52.2(49.0,55.4)$ & 179 & $20.1(17.5,22.8)$ \\
\hline Stage III & 0 & $0.0(0.0,0.0)$ & 140 & $40.1(35.0,45.3)$ & 56 & $32.7(25.7,39.8)$ \\
\hline
\end{tabular}

Table 3 should be replaced with the following:

Table 3. Surgical quality among women who underwent surgical resection for invasive breast cancer, Manitoba, $2010-2015$.

\begin{tabular}{|c|c|c|c|c|c|c|}
\hline \multirow[t]{2}{*}{ Characteristic } & \multicolumn{2}{|c|}{$\begin{array}{l}\text { Axillary Lymph Node Dissection } \\
\text { for Node Negative Disease } \\
\qquad(n=2379)\end{array}$} & \multicolumn{2}{|c|}{$\begin{array}{l}\leq 30 \text { Days Between First Surgical } \\
\text { Consult and First Surgery } \\
\qquad(n=2526)\end{array}$} & \multicolumn{2}{|c|}{$\begin{array}{c}\text { Re-excision After } \\
\text { Breast-Conserving Surgery } \\
(n=2439)\end{array}$} \\
\hline & $n$ & $\%(95 \%$ CI $)$ & $n$ & $\%(95 \%$ CI $)$ & $n$ & $\%(95 \%$ CI $)$ \\
\hline Manitoba & 137 & 5.8 & 1245 & 49.3 & 450 & 18.5 \\
\hline \multicolumn{7}{|c|}{ Age Group } \\
\hline $20-39$ & - & $1.9(0.0,5.7)$ & 27 & $32.9(22.8,43.1)$ & 20 & $35.7(23.2,48.3)$ \\
\hline $40-49$ & - & $6(3.1,9.0)$ & 176 & $49.4(44.2,54.6)$ & 70 & $24.1(19.1,29.0)$ \\
\hline $50-59$ & - & $3.8(2.2,5.4)$ & 299 & $46.7(42.9,50.6)$ & 118 & $20.0(16.8,23.2)$ \\
\hline $60-69$ & - & $5.4(3.8,7.0)$ & 409 & $54.0(50.4,57.5)$ & 133 & $17.6(14.9,20.3)$ \\
\hline $70-79$ & - & $7.3(5.0,9.6)$ & 227 & $48.6(44.1,53.1)$ & 76 & $15.7(12.5,19.0)$ \\
\hline $80+$ & - & $7.9(4.9,10.9)$ & 107 & $48.0(41.4,54.5)$ & 33 & $12.5(8.5,16.5)$ \\
\hline \multicolumn{7}{|c|}{ RHA of Residence (at diagnosis) } \\
\hline Urban & - & $3.0(2.1,3.9)$ & 766 & $47.9(45.5,50.4)$ & 258 & $17.0(15.1,18.9)$ \\
\hline Rural 1 & - & $15.9(12.0,19.8)$ & 160 & $60.2(54.3,66.0)$ & 73 & $23.5(18.8,28.2)$ \\
\hline Rural 2 & - & $8.0(4.9,11.2)$ & 151 & $49.2(43.6,54.8)$ & 66 & $24.6(19.5,29.8)$ \\
\hline Rural 3 & - & $4.8(2.3,7.4)$ & 150 & $49.8(44.2,55.5)$ & 42 & $14.7(10.6,18.8)$ \\
\hline Rural 4 & - & $7.1(0.4,13.9)$ & 18 & $33.3(20.8,45.9)$ & 11 & $19.3(9.1,29.5)$ \\
\hline
\end{tabular}


Table 3. Cont.

\begin{tabular}{|c|c|c|c|c|c|c|}
\hline \multirow[t]{2}{*}{ Characteristic } & \multicolumn{2}{|c|}{$\begin{array}{l}\text { Axillary Lymph Node Dissection } \\
\text { for Node Negative Disease } \\
(n=2379)\end{array}$} & \multicolumn{2}{|c|}{$\begin{array}{l}\leq 30 \text { Days Between First Surgical } \\
\text { Consult and First Surgery } \\
\qquad(n=2526)\end{array}$} & \multicolumn{2}{|c|}{$\begin{array}{c}\text { Re-excision After } \\
\text { Breast-Conserving Surgery } \\
(n=2439)\end{array}$} \\
\hline & $n$ & $\%(95 \% \mathrm{CI})$ & $n$ & $\%(95 \% \mathrm{CI})$ & $n$ & $\%(95 \% \mathrm{CI})$ \\
\hline \multicolumn{7}{|c|}{ RHA of Surgery } \\
\hline Urban 1 & 71 & $3.4(2.6,4.2)$ & \multicolumn{2}{|c|}{$\mathrm{n} / \mathrm{a}$} & 376 & $17.5(15.9,19.1)$ \\
\hline Rural 1 & 52 & $20.4(15.4,25.3)$ & \multirow{2}{*}{\multicolumn{2}{|c|}{$\mathrm{n} / \mathrm{a}$}} & 57 & $23.0(17.7,28.2)$ \\
\hline Rural 2 & 14 & $32.6(18.6,46.6)$ & & & & \\
\hline Rural 2-4 & $\mathrm{n} / \mathrm{a}$ & $\mathrm{n} / \mathrm{a}$ & $\mathrm{n} / \mathrm{a}$ & $\mathrm{n} / \mathrm{a}$ & 15 & $46.8(29.6,64.2)$ \\
\hline \multicolumn{7}{|c|}{ Stage } \\
\hline Stage I & 80 & $4.9(3.9,6.0)$ & 609 & $49.6(46.8,52.4)$ & 208 & $15.5(13.6,17.4)$ \\
\hline Stage II & 57 & $7.7(5.8,9.6)$ & 496 & $52.2(49.0,55.4)$ & 179 & $20.1(17.5,22.8)$ \\
\hline Stage III & 0 & $0.0(0.0,0.0)$ & 140 & $40.1(35.0,45.3)$ & 56 & $32.7(25.7,39.8)$ \\
\hline
\end{tabular}

$\mathrm{n} / \mathrm{a}$ : The RHA of surgery stratification is not applicable for some indicators.

In the original article, the discussion stated the following, "In our study, we found that $19.6 \%$ of women in Manitoba with node-negative cancer underwent an ALND; other studies from other jurisdictions found this number to be as high as $49 \%$ [36]. Therefore, Manitoba meets the minimum standard published by EUSOMA but not the target".

It should be replaced with the following,

"In our study, we found that $5.8 \%$ of women in Manitoba with node-negative cancer underwent an ALND, but that the number varied greatly by surgery geographic locations; other studies from other jurisdictions found this number to be as high as $49 \%$ [36]. Therefore, Manitoba meets the minimum standard published by EUSOMA as well as the target, but on a global scale, but some centers are falling well behind this benchmark".

The authors apologize for any inconvenience caused and state that the scientific conclusions are unaffected. The original article has been updated.

Conflicts of Interest: The authors declare no conflict of interest.

\section{Reference}

1. Ratnayake, I.; Hebbard, P.; Feely, A.; Biswanger, N.; Decker, K. Assessment of Breast Cancer Surgery in Manitoba: A Descriptive Study. Curr. Oncol. 2021, 28, 581-592. [CrossRef] [PubMed] 